\title{
Failure Modes in Retinal Detachment Surgery
}

\author{
Steve Charles \\ Charles Retina Institute, Memphis, TN, USA
}

T

here are many causes of failed retinal detachment surgery. This editorial will discuss techniques to improve outcomes and $r$ educe complications.

DOl: https://doi.org/10.17925/USOR.2019.12.1.17

\section{Keywords}

Retinal detachment, wide-angle visualization, vitreous traction, laser, silicone oil, retinopexy, PFO

Disclosure: Steve Charles has received consultant/ speaker/funding from Alcon Laboratories, Inc.

Review Process: Double-blind peer review.

Compliance with Ethics: This article is an opinion piece and does not report on new clinical data, or any studies with human or anima subjects performed by any of the authors.

Authorship: All named authors meet the International Committee of Medical Journal Editors (ICMJE) criteria for authorship of this manuscript, take responsibility for the integrity of the work as a whole, and have given final approval to the version to be published.

Received: March 14, 2019

Accepted: March 29, 2019

Citation: US Ophthalmic Review. 2019;12(1):17-8

Corresponding Author: Steve Charles, Charles Retina Institute, 1432 Kimbrough Road, Germantown, TN 38138, USA. E: scharles@att.net

Support: No funding was received in the publication of this article.
Poor visualization is a common cause of failed retinal detachment surgery, both because of failure to identify and treat retinal breaks and because of residual vitreoretinal traction (see Figure 1). Cataract, especially cortical and posterior subcapsular cataract, limits crucial visualization of the peripheral retina. ${ }^{1,2}$ Combined phacoemulsification and vitrectomy are commonly performed outside the USA, but often yield refractive surprises and a higher incidence of posterior synechia and intraocular lens (IOL) decentration. Additionally, combined procedures can cause intraoperative miosis requiring iris hooks, which increase inflammation. If already pseudophakic, posterior capsular opacification (PCO) can limit visualization, but surgical capsulotomy invariably results in IOL fogging. ${ }^{3}$ Fogging is not caused solely by silicone IOLS, although they have a higher thermal mass and a greater incidence of PCO; acrylic and polymethylmethacrylate (PMMA) lens develop fogging as well if there is an open capsule and interrupted anterior vitreous cortex. If a yttrium aluminum garnet (YAG) laser capsulotomy has already been performed, the vitreoretinal surgeon should not remove an intact anterior vitreous cortex to help prevent IOL fogging. If IOL fogging occurs there are two options:

- The retina can be reattached with N-perfluoro-octane (PFO) injected with a dual-bore cannula and followed by PFO-gas exchange (25\% SF $)$ with a soft-tip cannula in the optic nerve cup; unfortunately, residual PFO droplets often occur.

- Alternatively, fluid-air exchange followed byair-siliconeoilexchangeand laserendophotocoagulation to all retinal breaks after the oil is in place. Internal drainage of subretinal fluid through existing retinal breaks or a drainage retinotomy can be accomplished without difficulty "under" oil. A full oil fill is especially critical if there are inferior retinal breaks. The oil can be removed in a month or so.

Conceptualization is critical to visualization. It is often useful to ask the patient where in the visual field shadow (field defect) was first noted. The highest point of the detachment can also suggest where to look for retinal breaks. Concentric demarcation lines can also indicate where the detachment began. Localized retinal pigment epithelium (RPE) hyperpigmentation often occurs at the site of retinal breaks because the RPE responds to trans-hole fluid flow by elongation of apical processes to increase surface area for the RPE fixed pressure gradient pump. Melanin migration into the apical processes explains the clinical appearance of hyperpigmentation. The terminal ends of lattice degeneration is a common site of retinal breaks. Round, so-called atrophic, retinal holes or operculated holes can cause retinal detachment. Clinically, these holes can be observed but all retinal defects must be treated when vitrectomy is performed because the viscosity is permanently reduced by 800 -fold. Sub-clinical detachments virtually never occur after vitrectomy, but rather, a very small hole typically causes a rapid total retinal detachment because of the reduced viscosity.

The initiation of core vitrectomy should be thought of as a diagnostic tool, not just a procedural step, as subretinal fluid often flows from retinal breaks, causing an optical effect due to admixture of two fluids of different indices of refraction (Schlieren). This typically occurs with low levels of linear (proportional) vacuum while removing vitreous at the posterior edge of the vitreous base. Focal and retro-illumination with the endoilluminator (not chandelier) often allows identification of collagen fiber bundles extending to the flap of a flap tear (horseshoe tear). Chandelier illumination while performing scleral depression is critical to visualization of retinal breaks. The primary advantage is the ability to view equatorial retina in contour rather than en face. In addition, the periphery can be moved inwards 
Figure 1: Causes of failed retinal detachment surgery

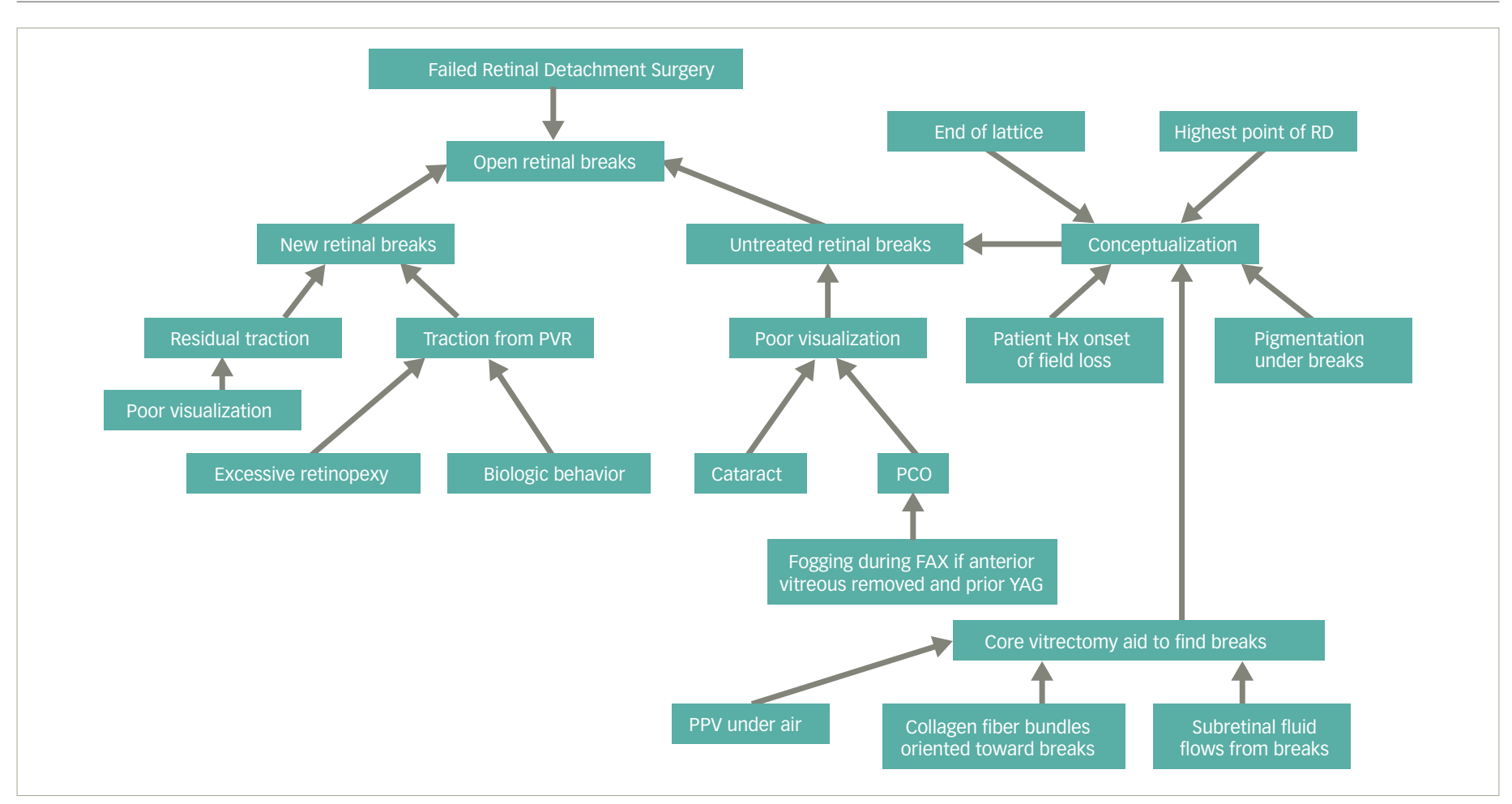

FAX = fluid-air exchange; $H X=$ history; $P C O=$ posterior capsular opacification; $P V R=$ proliferative vitreoretinopathy; $R D=$ retinal detatchment; $Y A G=y$ ttrium aluminum garnet.

using scleral depression when cortical cataract, retained cortex, or PCO limits peripheral visualization.

For decades, the author has advocated vitrectomy "under" air ${ }^{4}$ as well as "under" oil to identify residual vitreoretinal traction; a common cause of failed retinal detachment surgery. After identifying all retinal breaks and resolving all apparent traction, trans-break or -retinotomy drainage of subretinal fluid is initiated. After the retina either becomes reattached (typically with a single break) or subretinal fluid stops decreasing (multiple breaks) fluid-air exchange is initiated while continuing internal drainage of subretinal fluid. The author refers to this concept as the "reattachment experiment". Residual vitreous traction (epiretinal membranes, subretinal bands or need for retinectomy) can often be identified at this stage because of specular reflection (sheen) and refractive effects and vitreous traction removed "under" air. ${ }^{5}$ Similarly, residual vitreous traction can be identified and removed "under" PFO using what the author refers to as interface vitrectomy. ${ }^{6}$

Although many surgeons believe that a scleral buckle can mitigate residual traction and prevent surgical failure, combining a scleral buckle with vitrectomy is not the answer. A scleral buckle without retinopexy to every retinal break still results in failure. Although the putative rationale for adding a scleral buckle to vitrectomy is supporting the vitreous base; many (but not all) published series do not support the conclusion of better outcomes with scleral buckles and breaks often occur posterior to the buckle.-10 It is better to identify and remove vitreous traction than hope a scleral buckle will somehow take care of it. Encircling scleral buckles induce significant axial myopia, typically 2.75 diopters, which is completely unacceptable to patients that have had refractive surgery or refractive cataract surgery. ${ }^{11}$ scleral buckles increase phorias and tropias by $50 \%$, cause slight ptosis, cause ocular surface disorders because of poor closure of the $360^{\circ}$ peritomy, and make subsequent glaucoma procedures more difficult because of conjunctival adherence to Tenon's capsule and episclera. Postoperative pain is significantly worse than vitrectomy alone and infection/extrusion and intrusion of scleral buckles are potential long term complications. ${ }^{12}$

Medium-term PFO is ideal for inferior retinal detachments and obviates the need for a scleral buckle. ${ }^{13}$ Medium-term PFO will slowly and safely create a posterior vitreous detachment (PVD) during the 14 days the PFO is in place; ideal for young myopes without a PVD. A full fill with N-perfluoro-octane is crucial to reduce the formation of small bubbles. A dual bore cannula and the VFC with 8 psi pressure is ideal to inject the PFO; the cannula tip should be maintained at the top of the expanding bubble while injecting to produce a single bubble. Confluent laser is applied to all retinal breaks. Medium-term PFO patients can sit, stand, drive, work, and fly with no need to be face down. ${ }^{13}$

Performing multiple rows of $360^{\circ}$ laser in lieu of identifying and treating all retinal breaks is not the right answer. New retinal breaks typically occur at the posterior edge of prior retinopexy because retinopexy delimits retinal elasticity. Multiple rows of $360^{\circ}$ laser cause more inflammation (possibly increasing the rate of proliferative vitreoretinopathy [PVR], and cystoid macular edema [CME]) and increases the risk of lens damage by the laser probe in phakic eyes. Rows of laser posterior to the equator can produce a so-called "donut detachment" anterior to the rows of laser. These chronic retinal detachments produce vascular endothelial growth factor (VEGF) and therefore neovascularization on the retinal surface, iris, and trabecular meshwork. In addition, "donut detachments" are more likely to develop PVR and hypotony secondary to epiciliary tissue. Confluent laser is better than rows of spots. Moving the laser while applying energy evens out the 
thermal effect and makes the adherence between retina and RPE more uniform. Spots either overlap producing excessive thermal damage or "under-lap" leaving gaps for subretinal fluid leakage. Spots are an outdated concept in the operating room (as opposed to office laser) as they are historically based on diathermy and cryopexy probes which cannot be moved while energy is being applied.

Proliferative vitreoretinopathy (PVR) can occur spontaneously or develop as an iatrogenic result of excessive retinopexy. PVR should be thought of as a keloid, i.e. excessive healing. Operating on inflamed eyes instead of delaying until inflammation subsides is a common cause of PVR. Use of relaxing retinotomy ${ }^{14}$ instead of retinectomy leads to greater anterior PVR, epiciliary tissue, and hypotony. Performing lensectomy and leaving the capsule in anticipation of subsequent sulcus IOL placement is a significant and common error. The capsule always harbors residual lens epithelial cells which proliferate, causing what the author calls neocortex, inflammation, and adherence of capsule to iris. In addition to the concave iris configuration and fixed pupil, these patients often develop anterior loop traction due to residual peripheral vitreous becoming adherent to capsular fibrosis. Complete capsule removal with end-grasping forceps should always be performed to prevent the aforementioned phenomenon as well as fibrosis-related closure of inferior peripheral iridectomies in silicone oil cases.

Critical elements of successful surgery include use of wide-angle visualization and scleral depression, enabling removal of vitreous traction from all flap tears as well as anterior to all tears. Remove as much peripheral vitreous as possible. Never hesitate to remove the lens or IOL if peripheral visualization and traction removal is limited by peripheral visualization. Always remove the capsule en total if lensectomy is performed. Avoid combined phaco and vitrectomy. Repair all cases without scleral buckling. Perform moderate intensity confluent laser around retinal breaks and suspicious areas; do not perform multiple rows of interrupted laser. If the eye is significantly inflamed, use silicone oil but do not perform retinopexy until weeks or months later when the eye is no longer inflamed. Make use of medium-term PFO for inferior retinal detachments. $\square$
1. Sodhi A, Leung $L S, D o D V$, et al. Recent trends in the management of rhegmatogenous retinal detachment. Surv Ophthalmol. 2008:5:50-67.

2. Williamson TH, Lee EJ, Shunmugam M. Characteristics of rhegmatogenous retinal detachment and their relationship to success rates of surgery. Retina. 2014;34:1421-7.

3. Kuhn F, Aylward B. Rhegmatogenous retinal detachment: a reappraisal of its pathophysiology and treatment. Ophthal Res. 2014;51:15-31.

4. Rosengren B. Results of treatment of detachment of the retina with diathermy and injection of air into the vitreous. Acta Ophthalmol. 1938:16:573-9.

5. Tan HS, Oberstein SY, Mura M, Bijl HM. Air versus gas tamponade in retinal detachment surgery. Br J Ophthalmol. 2013;97:80-2.
6. Chang S, Lincoff $\mathrm{H}$, Zimmerman NJ, et al. Giant retinal tears. Surgical techniques and results using perfluorocarbon liquid. Arch Ophthalmol. 1989:107:761-6.

7. Escoffery RF, Olk RJ, Grand MG, Boniuk I. Vitrectomy without scleral buckling for primary rhegmatogenous retinal detachment. Am J Ophthalmol. 1985;99:275-81.

8. Siqueira RC, Gomes CV, Dalloul C, Jorge R. Vitrectomy with and without scleral buckling for retinal detachment. Arq Bras Oftalmol. 2007;70:298-302.

9. Stangos AN, Petropoulos IK, Brozou CG et al. Pars-plana vitrectomy alone vs vitrectomy with scleral buckling for primary rhegmatogenous pseudophakic retinal detachment. Am J Ophthalmol. 2004:138: 952-8.

10. Mendrinos E, Dang-Burgener NP, Stangos AN et al. Primary vitrectomy without scleral buckling for pseudophakic rhegmatogenous retinal detachment. Am J Ophthalmol. 2008;145:1063-70

11. Smiddy WE, Loupe DN, Michels RG et al. Refractive Changes After Scleral Buckling Surgery. Arch Oph. 1989:107:1469-71.

12. Roldan-Pallares M, del Castillo Sanz $J$, Awad-El Susi S, Refojo MF. Long-term complications of silicone and hydrogel explants in retinal reattachment surgery. Arch Oph. 1999;117;197-201

13. Sigler EJ, Randolph JC, Calzada Jl, et al. Pars plana vitrectomy with medium-term postoperative perfluoro-N-octane for recurrent inferior retinal detachment complicated by advanced proliferative vitreoretinopathy. Retina. 2013;33:1-7.

14. Machemer R. [Cutting of the retina: a means of therapy for retinal reattachment (author's transl)]. Klin Monbl Augenheilkd. 1979:175:597-601. 